\title{
Квантовые поправки к проводимости и аномальный эффект Холла в квантовых ямах InGaAs с пространственно отделенной примесью Mn
}

\author{
(С) Л.Н. Овешников ${ }^{1,2}$, Е.И. Нехаева ${ }^{1,2}$ \\ ${ }^{1}$ Национальный исследовательский центр „Курчатовский институт“, \\ 123182 Москва, Россия \\ ${ }^{2}$ Физический институт им. П.Н. Лебедева Российской академии наук, \\ 119991 Москва, Россия \\ E-mail: Oveshln@gmail.com
}

(Получена 20 декабря 2016 г. Принята к печати 28 декабря 2016 г.)

\begin{abstract}
Исследован магнетотранспорт в гетероструктурах с пространственным разделением магнитной примеси $\mathrm{Mn}$ и квантовой ямы InGaAs. Анализ наблюдаемого эффекта слабой локализации с помощью формулы Хиками-Ларкина-Нагаоки приводит к аномально низкому значению префактора (0.4). Полученные данные указывают на то, что основным механизмом сбоя фазы может быть неупругое $e-e$ рассеяние. Анализ проводимости и магнетосопротивления указывает на доминантную роль спин-зависимого рассеяния на флуктуациях магнитной подсистемы, что согласуется с увеличением амплитуды отрицательного магнетосопротивления и увеличением друдевской проводимости при охлаждении. Исследована природа аномального эффекта Холла в данных структурах, в частности присутствуют указания на наличие топологического вклада при низких температурах.
\end{abstract}

DOI: $10.21883 /$ FTP.2017.10.45014.8489

Ключевой идеей для дальнейшего развития спинтронных технологий является совмещение возможности управления электронным спином и богатого функционала полупроводниковых систем [1]. Таким образом, исследования в области полупроводниковой спинтроники являются одними из наиболее приоритетных для создания наноэлектроники следующего поколения. Большая часть подобных исследований связана с пленками разбавленного магнитного полупроводника ( $\mathrm{Ga}, \mathrm{Mn}) \mathrm{As}$ с трехмерным спектром носителей заряда [2]. Для данных систем были достигнуты значительные успехи в понимании и описании наблюдаемых явлений. В частности, были обозначены релевантные механизмы обменного взаимодействия между магнитными моментами атомов $\mathrm{Mn}$ в полупроводниковой матрице GaAs, продемонстрировано наличие спиновой поляризации свободных носителей заряда, обусловленной взаимодействием магнитной и проводящей подсистем, а также были сформулированы основные технологические особенности получения подобных структур [2]. Однако для потенциальной интеграции ферромагнитных (ФМ) полупроводниковых устройств в существующие технологии принципиально важным является исследование магнитных систем с двумерным спектром носителей заряда. Результаты исследований магнитных гетероструктур позволили частично выяснить особенности таких систем, в том числе, в случае пространственного разделения магнитных атомов Мn и двумерного дырочного газа, когда их взаимодействие является существенно непрямым [3-7]. Наличие этого взаимодействия должно оказывать заметное влияние на зарядовый транспорт в системе, однако природа наблюдаемых особен- ностей магнетотранспорта еще далека от детального понимания.

Важной частью характеризации неупорядоченных систем является исследование квантово-когерентных явлений [8]. Полагается, что в ФМ материалах явление слабой антилокализации подавляется за счет сильной спиновой поляризации носителей заряда, а внутреннее магнитное поле системы нарушает фазовую когерентность при интерференции в локализационном контуре, приводя к отсутствию эффекта слабой локализации (СЛ) [9]. Однако, в отличие от ФМ металлов, система ( $\mathrm{Ga}, \mathrm{Mn}) \mathrm{As}$ за счет сравнительно малой концентрации магнитных атомов является слабым ФМ. С одной стороны, это позволяет более подробно исследовать в ней особенности перехода „металл-диэлектрик“, а с другой - наличие слабой спиновой поляризации приводит к неоднозначной интерпретации экспериментальных данных [10-12].

Цель настоящей работы состоит в выявлении особенностей в поведении магнитотранспорта двумерных носителей заряда, возникающих вследствие их взаимодействия с магнитной примесью, пространственно отделенной от проводящего канала. В частности, исследованы особенности аномального эффекта Холла (АЭХ), являющегося следствием взаимодействия магнитной и проводящей подсистем. Также проанализированы эффект Шубникова-де-Гааза и эффект слабой локализации, наблюдаемые в области низких температур.

В работе измерялись температурные и магнитополевые зависимости компонент тензора сопротивлений $\left(\begin{array}{lll}R_{x x} & \text { и } R_{x y}\end{array}\right)$ гетроструктур с квантовой ямой (КЯ) $\mathrm{GaAs} / \mathrm{In}_{x} \mathrm{Ga}_{1-x} \mathrm{As}(x \approx 0.2)$ и с пространственно отде- 
ленной от нее магнитной примесью марганца, проявляющей акцепторные свойства. Образцы были выращены на подложках GaAs методом MOCVD (metalorganic chemical vapour deposition) с лазерным распылением Mn-мишени, после чего на поверхности образцов были сформированы мезаструктуры в виде холловских мостиков (с отношением длины канала к его ширине $\approx 5)$. Структура исследованных образцов представлена в $[4,13]$. Ввиду диффузии атомов марганца в процессе роста структуры формируется примесный слой $(\mathrm{Ga}, \mathrm{Mn}) \mathrm{As}$ толщиной $3-5 \mathrm{HM}$, отделенный от квантовой ямы спейсером GaAs толщиной 3 нм. В результате неравномерного распределения атомов марганца в плоскости образца возникает крупномасштабный флуктуационный потенциал [5], определяющий характер проводимости структуры. Исследуемые образцы 4843 и 4831 отличаются содержанием примеси $\mathrm{Mn}-0.4$ и 0.5 монослоя $\left(\approx 1.3\right.$ и $\left.1.6 \cdot 10^{14} \mathrm{~cm}^{-2}\right)$ соответственно. Пространственное разделение примесного слоя, содержащего большое число рассеивающих центров, и квантовой ямы увеличивает подвижности дырок в проводящем канале [4], сохраняя при этом их обменное взаимодействие с магнитными моментами Mn. Подвижности и концентрации дырок в исследуемых образцах $\left(\mu_{\mathrm{H}}\right.$ и $\left.p_{\mathrm{H}}\right)$, определенные из нормального эффекта Холла, оказались достаточно высокими, что указывает на дрейфовый характер проводимости в канале, и при $T \approx 4 \mathrm{~K}$ составили $\approx 2100$ и $1900 \mathrm{~cm}^{2} /(\mathrm{B} \cdot \mathrm{c})$ и 0.96 и $0.72 \cdot 10^{12} \mathrm{~cm}^{-2}$ для образцов 4843 и 4831 соответственно. При этом полученные $\mu_{\mathrm{H}}$ более чем на порядок превосходят характерные значения для пленок ( $\mathrm{Ga}, \mathrm{Mn}) \mathrm{As}$ с трехмерным спектром носителей заряда, для которых обычно $\mu \leq 10 \mathrm{~cm}^{2} /(\mathrm{B} \cdot \mathrm{c})$. Из-за сравнительно слабого обменного взаимодействия делокализованных дырок в квантовой яме и магнитных моментов $\mathrm{Mn}$, т.е. проводящей и магнитной подсистем, исследуемые гетероструктуры можно рассматривать как обычные квантовые ямы, возмущенные присутствием магнитного слоя. Это позволяет выделить особенности зарядового транспорта, связанные именно с наличием магнитной примеси.

Поскольку пространственное распределение атомов Mn неоднородно, то и распределение связанных с ними магнитных моментов также оказывается неоднородным. В результате в образце присутствуют области локального магнитного упорядочения. При понижении температуры эти области начинают увеличиваться в размере [14] и взаимодействовать, и при критической температуре $T=T_{c}$ формируется бесконечный перколяционный магнитный кластер [15]. Вблизи этого перехода плотность критических флуктуаций оказывается велика. Ввиду рассеяния носителей заряда на этих флуктуациях на зависимости $\rho_{x x}(T)$ (см. рис. 1) появляется особенность, а непосредственно вблизи перехода на температурной зависимости производной $d \rho_{x x} / d T$ наблюдается отчетливый пик (см. вставку на рис. 1). По положению этого пика была оценена

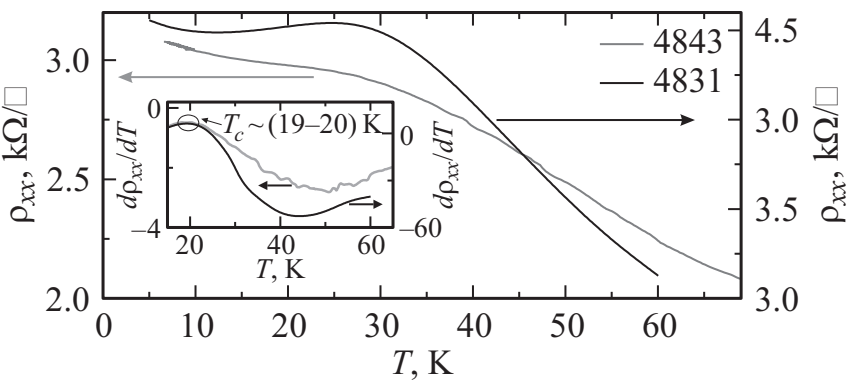

Рис. 1. Температурные зависимости сопротивления образцов 4843 (серые линии) и 4831 (черные линии); на вставке - температурная зависимость производной $d \rho_{x x} / d T$ с отмеченным значением критической температуры $T_{c}$.

$T_{c}$ [16], которая для исследуемых образцов составила $19-20 \mathrm{~K}$. Наличие данного пика является первичным свидетельством взаимодействия дырок в канале и магнитного слоя. Стоит отметить, что при дальнейшем понижении температуры (ниже $15 \mathrm{~K}$ ) $\rho_{x x}$ вновь начинает возрастать, что связывается с проявлением квантовых поправок к проводимости, рассматриваемых далее.

Магнитное упорядочение моментов Мn в подобных системах достигается за счет косвенного обмена, состоящего из внутрислоевого вклада [6], обусловленного носителями в связанных состояниях в примесном слое $(\mathrm{Ga}, \mathrm{Mn}) \mathrm{As}$, и вклада непрямого обменного взаимодействия [3], связанного с туннелированием делокализованных дырок из КЯ в примесный слой. Стоит отметить, что непрямой обмен может оказаться определяющим за счет его резонансного усиления при совпадении энергии дырок в канале с энергией связанных состояний в примесном слое [3]. Поскольку упомянутые механизмы обмена, аналогично механизму РККИ, имеют осциллирующий в пространстве характер, основное состояние магнитной подсистемы (в нулевом магнитном поле) в данных структурах является неколлинеарным.

На рис. 2 приведены магнитополевые зависимости сопротивления образцов при различных температурах. Для обоих образцов при $T<30 \mathrm{~K}$ магнетосопротивление (МC) становится отрицательным и в области низких температур наблюдаются осцилляции Шубникова-деГааза (ШдГ) (рис. 2,c). Частота наблюдаемых осцилляций ШдГ, определенная по их фурье-спектру (рис. 2, $d$ ), практически не зависит от температуры. Из полученных значений частот осцилляций были определены концентрации $p_{\mathrm{SdH}}$ двумерных дырок в канале, составившие 0.82 и $0.72 \cdot 10^{12} \mathrm{~cm}^{-2}$ для образцов 4843 и 4831 соответственно. Сравнивая полученные значения с приведенными ранее холловскими концентрациями, получается, что для образца 4831 наблюдается хорошее совпадение, а для образца 4843 - отличие $\approx 15 \%$, которое, скорее всего, связано с заполнением второй 

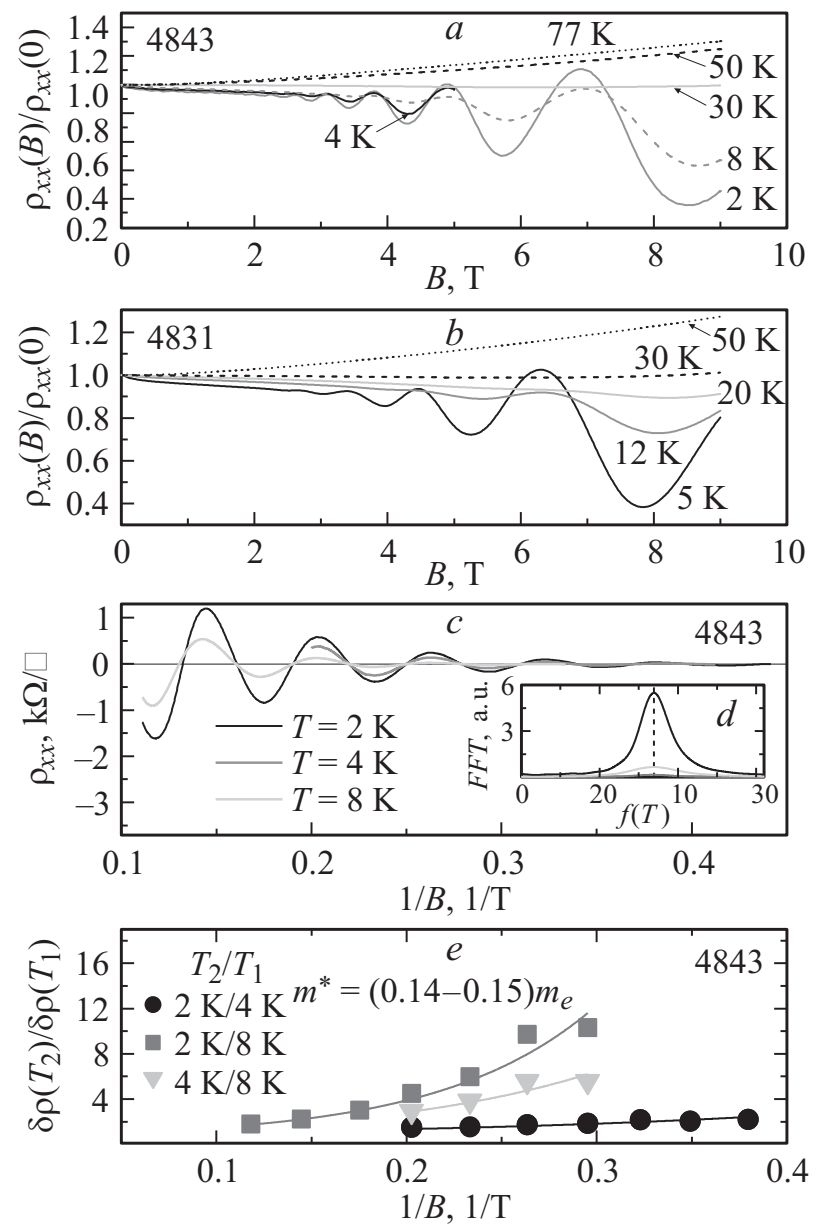

Рис. 2. Магнетосопротивление образцов 4843 (a) и 4831 (b) при различных температурах; $c-$ осцилляции ШдГ для образца 4843 после вычитания монотонной части магнетосопротивления; $d-$ фурье-спектр полученных осцилляций; $e$ - отношения амплитуд осцилляций ШдГ при различных температурах (точки) для образца 4843 как функции обратного магнитного поля и аппроксимации по формуле (1) (линии).

подзоны размерного квантования, не проявившейся в осцилляциях.

По амплитудам осцилляций $\delta \rho$ были определены эффективные массы $m^{*}$ носителей заряда в канале. Для этого по аналогии с [17] использовалось стандартное выражение для отношения амплитуд осцилляций ШдГ при $T_{1}>T_{2}$ :

$$
\frac{\delta \rho\left(T_{2}\right)}{\delta \rho\left(T_{1}\right)}=\frac{T_{2}}{T_{1}} \frac{\sinh \left(\Lambda\left(T_{1}\right)\right)}{\sinh \left(\Lambda\left(T_{2}\right)\right)}
$$

в котором $m^{*}$ является единственным подгоночным параметром (рис. 2,e), а коэффициент теплового затухания осцилляций $\Lambda$ задается следующей формулой:

$$
\Lambda\left(T_{i}\right)=\frac{2 \pi^{2} k_{\mathrm{B}} T_{i}}{\hbar \omega_{c}}
$$

где $k_{\mathrm{B}}-$ постоянная Больцмана, $\hbar-$ приведенная постоянная Планка, $\omega_{c}=e B / m^{*}, e-$ заряд электрона,
$B$ - магнитное поле. Стоит отметить, что при таком способе обработки осцилляций ШдГ точность определения $m^{*}$ задается количеством наблюдаемых пиков осцилляций. Поэтому в ряде случаев используемый подход оказывается предпочтительнее, чем определение $m^{*}$ по температурной эволюции одного пика, для которого требуется исследовать осцилляции ШдГ при значительно большем количестве температур. Полученные значения $m^{*} \approx 0.14-0.15 m_{e}\left(m_{e}-\right.$ масса свободного электрона) хорошо согласуются с литературными данными для аналогичных структур без магнитной примеси $[18,19]$. Таким образом, флуктуационный потенциал магнитной примеси не приводит к существенному изменению $m^{*}$.

Как уже отмечалось ранее, флуктуационный потенциал акцепторной примеси $\mathrm{Mn}$, имеющий кулоновскую природу, может индуцировать переход „металлдиэлектрик“ в проводящем канале при понижении концентрации носителей заряда. Поэтому в исследуемых структурах могут оказаться существенными квантовые поправки проводимости, чье исследование может выявить особенности, связанные с наличием магнитной подсистемы. О релевантности квантовых поправок в рассматриваемом случае свидетельствует отмеченный ранее рост сопротивления при $T<15 \mathrm{~K}$ и наличие резкого отрицательного МС в области слабых магнитных полей $(B<0.5$ Тл), связанного с эффектом СЛ. Магнетополевая зависимость интерференционных поправок в диффузионном режиме обычно описывается формулой Хиками-Ларкина-Нагаоки (ХЛН) [20], которая может быть представлена в следующей форме:

$$
\frac{\Delta\left(1 / \rho_{x x}(B)\right)}{G_{0}}=\alpha\left[\psi\left(\frac{1}{2}+\frac{\hbar}{4 e l_{\phi}^{2} B}\right)-\ln \left(\frac{\hbar}{4 e l_{\phi}^{2} B}\right)\right],
$$

где $\psi$ - дигамма-функция, $l_{\phi}=\sqrt{D \tau_{\phi}}$ и $\tau_{\phi}$ - длина и время дефазировки соответственно, а $D=v_{F}^{2} \tau / 2$. В таком виде формула (3) содержит два подгоночных параметра (префактор $\alpha$ и $l_{\phi}$ ) и две физические константы. При аппроксимации экспериментальных данных этой формулой фактически подбирается ширина $\left(l_{\phi}\right)$ и высота $(\alpha)$ исследуемого участка магнетосопротивления, что приводит к достаточно точным результатам. Точность определения $\alpha$ и $l_{\phi}$ существенно зависит от количества точек, по которым идет аппроксимация. При этом в настоящей работе магнетосопротивление в области слабых магнитных полей было измерено с малым шагом, достаточным для установления формы кривой, что и позволило надежно определить величины $\alpha$ и $l_{\phi}$. Характеристическим параметром СЛ, определяющим область применимости формулы ХЛН, является величина транспортного поля $B_{t r}=\hbar /\left(2 e l^{2}\right)$, в котором эффект оказывается существенно подавлен. Для исследуемых образцов $B_{t r} \approx 0.3-0.5$ Тл. 

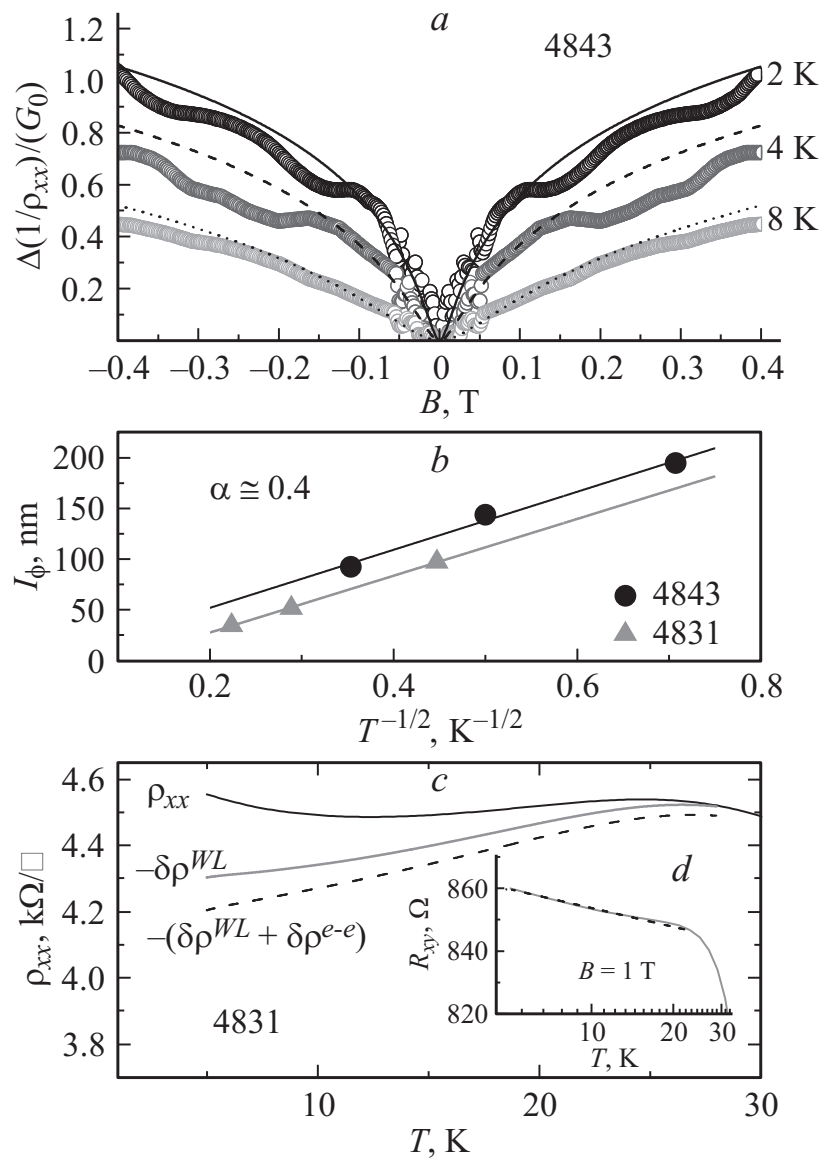

Рис. 3. $a-$ магнитополевая зависимость $\Delta\left(1 / \rho_{x x}(B)\right)=$ $=1 / \rho_{x x}(B)-1 / \rho_{x x}(0)$ в единицах $G_{0}=e^{2} /\left(2 \pi^{2} \hbar\right)$ для образца 4843 при различных температурах, точки - экспериментальные данные, линии - аппроксимация по формуле (3); $b$ - температурная зависимость длины дефазировки $l_{\phi}$; $c$ - температурная зависимость полного сопротивления $\rho_{x x}$ образца 4831, а также зависимости, полученные после вычета вкладов СЛ и $e-e$ взаимодействия (см. текст); $d-$ температурная зависимость полного холловского сопротивления образца 4831 в поле 1 Тл; пунктирной линией отмечено, что низкотемпературный участок имеет логарифмический характер.

Как показано на рис. 3, $a$, формулой ХЛН удается удовлетворительно описать экспериментальные данные в области $\pm(0.3-0.4) B_{t r}$, в которой проводилась аппроксимация. Для обоих образцов получается, что зависимость $l_{\phi}\left(T^{-1 / 2}\right)$ оказывается близка к линейной (рис. $3, b$ ), обычно это соответствует доминантной роли неупругого $e-e$ рассеяния в качестве основного механизма сбоя фазы. При этом длина свободного пробега дырок $l$ остается практически постоянной. Подобная картина наблюдается для большинства двумерных гетероструктур. Важной особенностью исследуемых образцов является аномально низкое значение префактора $\alpha \approx 0.4$. Отличие префактора от теоретического значения $\alpha=1$ в общем случае может быть связано с рядом причин [21], однако такое малое значение $\alpha$ сложно объяснить в рамках одной из них. Важно отметить, что для однозначного определения механизма фазовой релаксации в данных структурах желательно проводить исследования в более широком диапазоне температур и с меньшим шагом. Однако даже из полученных данных видно, что $l_{\phi}$ обладает выраженной температурной зависимостью, что отличается от поведения, предсказываемого для случая фазовой релаксации носителей заряда на ионах примеси или магнитных моментах, для которых $l_{\phi} \propto T^{0}$. Крайне важный результат данной части работы заключается в том, что на кривых магнетосопротивления не проявляется эффект слабой антилокализации. Как уже говорилось ранее, в ФМ системах эффект антилокализации должен быть подавлен, хотя в рассматриваемых структурах взаимодействие магнитной и проводящей подсистем достаточно слабое. Отсутствие эффекта антилокализации помогло избежать противоречий в интерпретации результатов, которые отмечались в ряде работ [10-12].

Пользуясь полученными значениями $l_{\phi}$ и $\alpha$, была рассчитана температурная зависимость поправки к проводимости $\delta \sigma^{W L}$, обусловленной СЛ:

$$
\delta \sigma^{W L}=-2 \alpha G_{0} \ln \left(\frac{l_{\phi}}{l}\right) .
$$

Экстраполируя полученную зависимость $\delta \sigma^{W L}(T)$ в область больших температур, была получена зависимость, приведенная на рис. 3, $c$ (серая кривая). Существенным является то, что полученная зависимость соответствует понижению сопротивления при охлаждении (металлический ход).

Расчет поправки $\delta \sigma^{e-e}$, связанной с $e-e$ взаимодействием в диффузионном канале [8], обычно осуществляется по формуле

$$
\delta \sigma^{e-e}=K_{e e} G_{0} \ln \left(\frac{k_{\mathrm{B}} T \tau}{\hbar}\right),
$$

где $T$ - температура, $\tau$ - время упругой релаксации импульса дырок в канале. При этом ключевой параметр $K_{e e}$ (префактор), связанный с ферми-жидкостным параметром взаимодействия, может определяться несколькими способами из эксперимента. В данной работе $K_{e e}$ определялось из температурной зависимости холловского сопротивления [22]. На рис. $3, d$ представлена зависимость $R_{x y}(T)$ для образца 4831 в поле 1 Тл (в котором эффект СЛ уже сильно подавлен). Как видно из рисунка, в области низких температур наблюдается участок логарифмического роста $R_{x y}(T)$, при этом из представленных ранее осцилляций ШдГ известно, что концентрация дырок фактически не зависит от температуры. Таким образом, можно полагать, что наблюдаемое поведение связано с эффектом $e-e$ взаимодействия, которому соответствует $d R_{x y} / d T<0$ при $k_{\mathrm{B}} T \tau / \hbar<1$, что справедливо в рассматриваемом интервале температур. 
Пользуясь соотношением $[8,22]$

$$
\frac{\delta R_{\mathrm{H}}}{R_{\mathrm{H}}}=-2 \frac{\delta \sigma_{e-e}}{\sigma_{\mathrm{D}}},
$$

где $\sigma_{\mathrm{D}}-$ друдевская проводимость, была оценена величина $K_{e e} \approx 0.2$. При этом использовалась приведенная на рис. 3,d температурная зависимость $R_{x y}(B=1$ Тл $) \approx R_{\mathrm{H}}$, а $\sigma_{\mathrm{D}}$ принималась равной обратной величине сопротивления системы после вычета вклада СЛ (серая кривая на рис. 3,c). Полученное значение $K_{e e}$ использовалось для расчета $\delta \sigma^{e-e}$ из формулы (5). В результате была получена температурная зависимость сопротивления образца 4831 без учета вкладов СЛ и $e-e$ взаимодействия (пунктирная кривая на рис. $3, c$ ).

Положительное значение $d \rho_{x x} / d T$ сохраняется даже без учета вклада, связанного с $e-e$ взаимодействием, а только при вычитании вклада, обусловленного эффектом СЛ. Поэтому неточность в определении $K_{e е}$ качественно не влияет на полученный результат. При этом оценочное значение $\delta \sigma^{e-e}$ заметно меньше проводимости всей системы (не более $1-2 \%$ ), а значит, наблюдаемое в эксперименте отрицательное МС, в монотонной части достигающее $\approx 20 \%$, не связано с $e-e$ взаимодействием.

Таким образом, наблюдаемое при $T<30 \mathrm{~K}$ отрицательное МС, скорее всего, связано с особенностями спин-зависимого рассеяния по аналогии с эффектом гигантского МС, что качественно согласуется с полученной температурной зависимостью сопротивления. Источником такого рассеяния могут служить флуктуации магнитной подсистемы, а также неколлинеарность ее основного состояния. Приложение внешнего магнитного поля приводит к переориентации магнитных моментов, подавлению флуктуаций и дополнительной спиновой поляризации дырок, за счет эффекта Зеемана. В свою очередь уменьшение температуры приводит к уменьшению плотности флуктуаций, а также увеличению „жесткости“ магнитной подсистемы и ее связи с делокализованными дырками в канале.

Заключительной частью настоящей работы является анализ аномального эффекта Холла (АЭХ) в данных структурах. Классически магнитополевую зависимость АЭХ связывают с изменением намагниченности М системы [23]. Тогда полное холловское сопротивление двумерной системы может быть представлено в виде следующей суммы:

$$
R_{x y}=R_{\mathrm{H}} \mathbf{B}+\mu_{0} R_{a} \mathbf{M},
$$

где $R_{\mathrm{H}}$ и $R_{a}$ - константы нормального и аномального эффектов Холла соответственно, $\mu_{0}$ - магнитная постоянная. Линейный наклон $R_{x y}(B)$ в области насыщения намагниченности (для данных образцов это соответствует $B>2$ Тл) дает величину $R_{\mathrm{H}}$, из которой определялись величины $p_{\mathrm{H}}$ и $\mu_{\mathrm{H}}$, приведенные ранее. На рис. $4, a$ приведены зависимости $R_{x y}(B)$ для образца 4831 при различных температурах. Характер зависимости $R_{x y}$ от температуры уже был показан на рис. $3, d$. Вычитанием
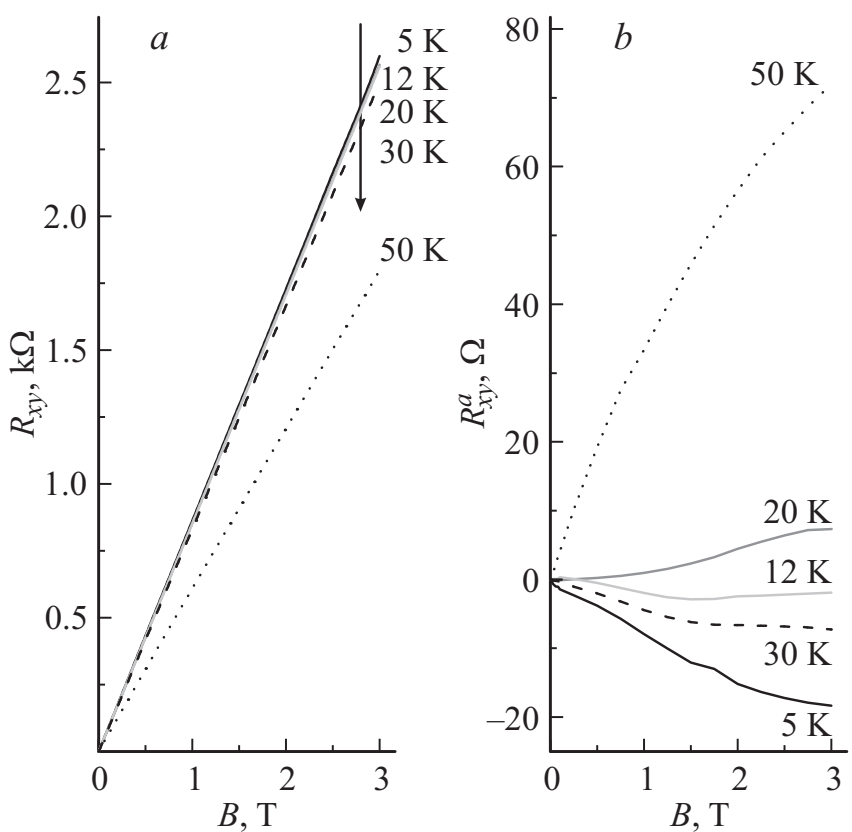

Рис. 4. Магнитополевые зависимости полного холловского сопротивления $(a)$ и его аномальной компоненты $(b)$ для образца 4831 при различных температурах.

линейной компоненты были получены магнетополевые зависимости АЭХ (рис. 4, $b$ ). Погрешность в измерении $R_{x y}$ составляет не более $0.1 \%$. При этом АЭХ в области слабых полей определяется с точностью не хуже $30 \%$, и не хуже $10 \%$ в магнитных полях выше 2 Тл. Как видно из рисунка, аномальное холловское сопротивление $R_{x y}^{a}$ оказывается достаточно малым, что доказывает справедливость использования полного холловского сопротивления для оценки $\delta \sigma^{e-e}$.

Наиболее распространенным способом исследования природы АЭХ является анализ параметрической зависимости аномальной компоненты холловского сопротивления $R_{x y}^{a}$ от сопротивления образца $\rho_{x x}$. Для рассматриваемого случая дрейфовой проводимости в литературе представлено три основных механизма АЭХ: бездиссипативный внутренний (intrinsic) механизм, связанный с фазой Берри, приобретаемой квазичастицей при движении в импульсном пространстве [24]; асимметричное рассеяние (skew-scattering), определяемое зависимостью направления траектории рассеянной квазичастицы от ее спина [25]; боковое смещение (sidejump), обусловленное возможностью неклассического сдвига траектории рассеянной квазичастицы в перпендикулярном ей направлении [26]. Вклад асимметричного рассеяния характеризуется линейной параметрической зависимостью $\left(R_{x y}^{a} \propto \rho_{x x}\right)$, а вклады двух других механизмов - квадратичной $\left(R_{x y}^{a} \propto \rho_{x x}^{2}\right)$. Кроме того, в данных системах возможно проявление топологического вклада в АЭХ [27-29], поскольку отсутствие жесткого ФМ упорядочения допускает возникновение нетривиальной топологии магнитной подсистемы в основном 
состоянии при понижении температуры. В данном контексте это соответствует формированию скирмионов (или аналогичных образований) за счет взаимодействия Дзялошинского-Мории. Топологический вклад в АЭХ в подобных системах был впервые экспериментально обнаружен в работе [13], что связывается с возникновением киральных магнитных конфигураций в слое Mn. В структурах, изученных в [13], в области низких температур наблюдался переход к прыжковой проводимости, в условиях которой топологический вклад успешно объясняется в рамках адиабатического приближения, т.е. при адиабатическом (обменном) взаимодействии носителей заряда в двумерном канале с киральной текстурой магнитной подсистемы. Однако в случае дрейфовой проводимости в канале, изучаемом в данной статье, поведение топологического вклада существенно зависит от выполнения или невыполнения условия адиабатичности взаимодействия двумерных дырок и киральных образований $[27,29]$. Поэтому количественное и качественное описание топологического вклада в этом случае затруднено отсутствием точной теории. Тем не менее благодаря формированию конфигураций намагниченности с ненулевой киральностью в слое $\mathrm{Mn}$ [13] топологический вклад должен иметь место и в рассматриваемых системах.

На рис. 5, $а$ приведена параметрическая зависимость $R_{x y}^{a}\left(\rho_{x x}\right)$ в поле 3 Тл для образца 4831. Стоит отметить, что АЭХ в данном образце наблюдается при температурах существенно выше $T_{c}$, т.е. в области локального магнитного упорядочения. Поскольку на $\rho_{x x}(T)$ присутствует локальный максимум (рис. 1), зависимость $R_{x y}^{a}\left(\rho_{x x}\right)$ становится неоднозначной функцией своего аргумента. Поэтому низкотемпературная часть кривой была графически отражена относительно пика $\rho_{x x}$ (рис. 5,a). В таком представлении кривая соответствует понижению температуры (слева-направо). Как видно из рисунка, высокотемпературная часть кривой характеризуется квадратичной зависимостью. Вблизи критической области появляется дополнительный положительный вклад с линейной параметрической зависимостью, который начинает быстро пропадать при охлаждении ниже $T_{c}$. В области низких температур кривая снова демонстрирует квадратичный спад. Таким образом, в некритических областях доминантный вклад в АЭХ вносит внутренний механизм и/или боковое смещение, а вблизи перехода в состояние с дальним магнитным упорядочением появляется дополнительный вклад, который, скорее всего, обусловлен асимметричным рассеянием дырок на критических флуктуациях магнитной подсистемы. В более слабых полях параметрические кривые имеют схожий характер (рис. $5, b$ и $c$ ), за исключением области низких температур $(T<9 \mathrm{~K})$. В слабом магнитном поле $(B=0.1-0.3$ Тл) на параметрической кривой появляется низкотемпературный участок выполаживания (flattening out), т.е. уменьшения значения производной $d R_{x y}^{a} / d \rho_{x x}$, как это проиллюстрировано на рис. 5, c серыми стрелками. Это подразумевает наличие
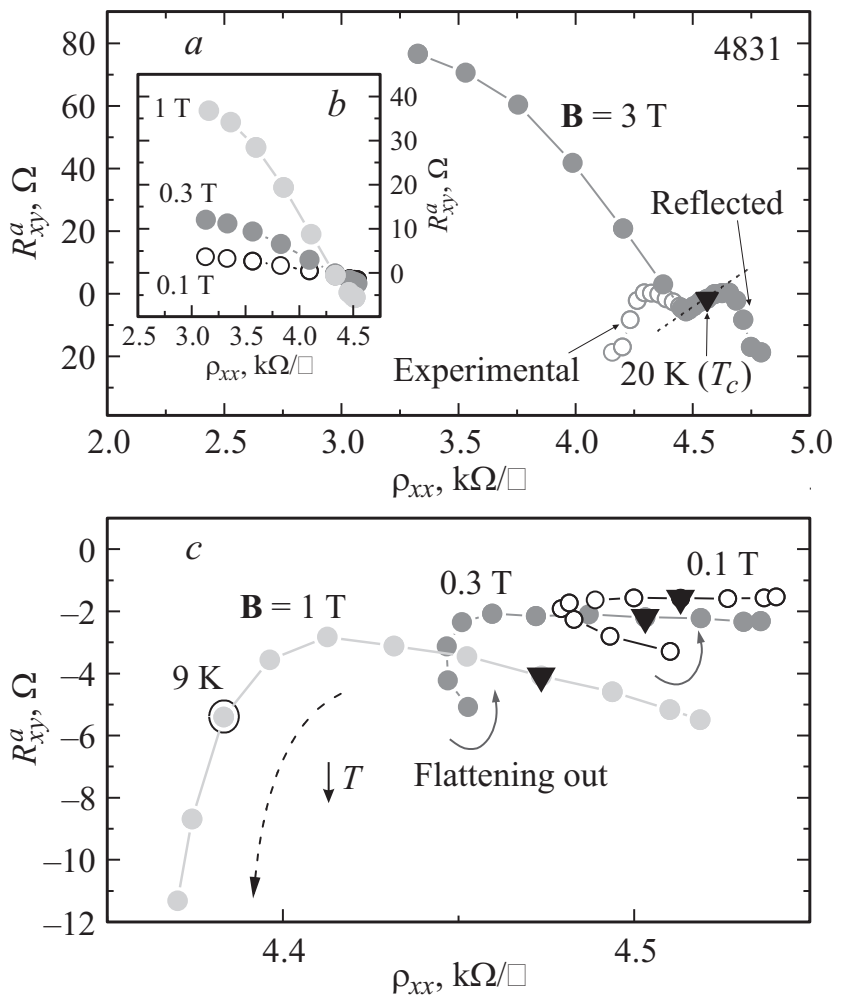

Рис. 5. Параметрические зависимости $\left(R_{x y}^{a}\left(\rho_{x x}\right)\right)$ для образца 4831 в поле 3 Тл $(a)$, кривые, полученные для меньших полей разбиты на интервалы высоких $(b)$ и низких температур $(c)$; черными треугольниками обозначено положение $T_{c}$; на $(a)$ проиллюстрировано графическое отражение кривой в области низких температур (см. текст); на (c) черным кругом отмечена точка, соответствующая температуре $9 \mathrm{~K}$, пунктирной стрелкой показано направление охлаждения, серыми стрелками показана тенденция к выполаживанию (flattening out) представленных зависимостей.

дополнительного положительного вклада. Как уже упоминалось ранее, это может быть связано с появлением нетривиальной топологии магнитной подсистемы, по аналогии с результатами работы [13].

Предположение о наличии вклада собственного механизма АЭХ в области низких температур, т. е. эффекта, связанного с фазой Берри, может частично объяснить аномальное значение префактора в формуле ХЛН. Например, в [30] было показано, что приобретение носителями заряда фазы Берри (которая в рассмотренном случае имела несколько другую природу) может влиять на форму МС, связанного со СЛ. С другой стороны, эффект СЛ может также влиять на АЭХ, например, как было показано в [31], это возможно через вклад асимметричного рассеяния, который в нашем случае подавлен при $T<9 \mathrm{~K}$. Таким образом, следует предположить, что наблюдаемое низкотемпературное выполаживание на параметрической зависимости АЭХ в нашем случае связано с другими вкладами АЭХ, а именно с топологическим вкладом. 
Таким образом, проведенный анализ магнетотранспорта в исследуемых системах указывает на ряд особенностей, связанных с наличием магнитной примеси Mn. Созданный ею флуктуационный потенциал приближает систему к переходу „металл-диэлектрик“, не приводя к существенному изменению эффективных масс дырок в канале. Установлено, что доминантным механизмом сбоя фазы в исследуемых структурах может быть неупругое $e-e$ рассеяние, как и в большинстве немагнитных гетероструктур. Заметным отличием является малое значение префактора, которое может быть связано с наличием магнитной подсистемы. Кроме того, наличие магнитного слоя приводит к появлению особенности на температурной зависимости сопротивления образцов и отрицательного МС, наблюдающегося при $T<30$ К вплоть до сильных магнитных полей. Главным отличием рассмотренных систем от тривиального случая является наблюдение АЭХ, который определяется вкладами разных механизмов. В частности, при $T<9 \mathrm{~K}$ присутствуют указания на наличие топологического вклада в АЭХ. Стоит отметить, что более подробное изучение этого вклада в исследуемых системах может пролить свет на детали поведения и особенности топологического механизма АЭХ за рамками адиабатического приближения.

Измерения при низких температурах в магнитных полях до 9 Тл были проведены в ЦКП ФИАН и ресурсном центре „Электрофизика“ в НИЦ „Курчатовский институт“. Авторы благодарят К.В. Меркутова за помощь в организации и проведении измерений и Б.Н. Звонкова за предоставление образцов. Также авторы благодарят Б.А. Аронзона, В.А. Кульбачинского, И.В. Рожанского и К.С. Денисова за ценные обсуждения и замечания по тексту статьи.

Работа выполнена при поддержке гранта РФФИ № 16-32-00446.

\section{Список литературы}

[1] S.A. Wolf, D.D. Awschalom, R.A. Buhrman, J.M. Daughton, S. von Molnar, M.L. Roukes, A.Y. Chtchelkanova, D.M. Treger. Science, 294, 1488 (2001).

[2] T. Jungwirth, J. Sinova, J. Masek, J. Kucera, A.H. MacDonald. Rev. Mod. Phys., 78, 809 (2006).

[3] I.V. Rozhansky, I.V. Krainov, N.S. Averkiev, B.A. Aronzon, A.B. Davydov, K.I. Kugel, V. Tripathi, E. Lahderanta. Appl. Phys. Lett., 106, 252402 (2015).

[4] B.A. Aronzon, M.V. Kovalchuk, E.M. Pashaev, M.A. Chuev, V.V. Kvardakov, I.A. Subbotin, V.V. Rylkov, M.A. Pankov, I.A. Likhachev, B.N. Zvonkov, Yu.A. Danilov, O.V. Vihrova, A.V. Lashkul, R. Laiho. J. Phys.: Condens. Matter, 20, 145207 (2008).

[5] V. Tripathi, K. Dhochak, B.A. Aronzon, V.V. Rylkov, A.B. Davydov, B. Raquet, M. Goiran, K.I. Kugel. Phys. Rev. B, 84, 075305 (2011).

[6] V.N. Men'shov, V.V. Tugushev, S. Caprara. Phys. Rev. B, 80, 035315 (2009).
[7] V.A. Kulbachinskii, P.V. Gurin, L.N. Oveshnikov. Nanosci. Nanotechnol. Lett., 4, 634 (2012).

[8] В.Ф. Гантмахер. Электроны в неупорядоченных средах (М., Физматлит, 2013).

[9] V.K. Dugaev, P. Bruno, J. Barnas. Phys. Rev. B, 64, 144423 (2001).

[10] D. Neumaier, K. Wagner, S. Geibler, U. Wurstbauer, J. Sadowski, W. Wegscheider, D. Weiss. Phys. Rev. Lett., 99, 116803 (2007).

[11] V.K. Dugaev, P. Bruno, J. Barnas. Phys. Rev. Lett., 101, 129701 (2008).

[12] D. Neumaier, K. Wagner, S. Geibler, U. Wurstbauer, J. Sadowski, W. Wegscheider, D. Weiss. Phys. Rev. Lett., 101, 129702 (2008).

[13] L.N. Oveshnikov, V.A. Kulbachinskii, A.B. Davydov, B.A. Aronzon, I.V. Rozhansky, N.S. Averkiev, K.I. Kugel, V. Tripathi. Sci. Rept., 5, 17158 (2015).

[14] V. Tripathi, K. Dhochak, B.A. Aronzon, B. Raquet, V.V. Tugushev, K.I. Kugel. Phys. Rev. B, 85, 214401 (2012).

[15] D.J. Priour, S. Das Sarma. Phys. Rev. B, 73, 165203 (2006).

[16] M. Wang, R.A. Marshall, K.W. Edmonds, A.W. Rushforth, R.P. Campion, B.L. Gallagher. Appl. Phys. Lett., 104, 132406 (2014).

[17] N.A. Yuzeeva, G.B. Galiev, E.A. Klimov, L.N. Oveshnikov, R.A. Lunin, V.A. Kulbachinskii. Phys. Procedia, 72, 425 (2015).

[18] G.M. Minkov, A.A. Sherstobitov, A.V. Germanenko, O.E. Rut, V.A. Larionova, B.N. Zvonkov. Phys. Rev. B, 71, 165312 (2005).

[19] J.E. Schirber, I.J. Fritz, L.R. Dawson. Appl. Phys. Lett., 46 (2), 187 (1985).

[20] S. Hikami, A.I. Larkin, Y. Nagaoka. Prog. Theor. Phys., 63 (2), 707 (1980).

[21] G.M. Minkov, A.V. Germanenko, I.V. Gornyi. Phys. Rev. B, 70, 245423 (2004).

[22] A.Yu. Kuntsevich, A.A. Gabdullin, V.A. Prudkogliad, Yu.G. Selivanov, E.G. Chizhevskii, V.M. Pudalov. Phys. Rev B, 94, 235401 (2016).

[23] E.M. Pugh, T.W. Lippert. Phys. Rev., 42, 709 (1932).

[24] T. Jungwirth, Q. Niu, A.H. MacDonald. Phys. Rev. Lett., 88 (20), 207208 (2002).

[25] J. Smit. Physica (Amsterdam), 24, 39 (1958).

[26] L. Berger. Phys. Rev. B, 2 (11), 4559 (1970).

[27] P. Bruno, V.K. Dugaev, M. Taillefumier. Phys. Rev. Lett., 93 (9), 096806 (2004).

[28] A. Neubauer, C. Pfleiderer, B. Binz, A. Rosch, R. Ritz, P.G. Niklowitz, P. Boni. Phys. Rev. Lett., 102, 186602 (2009).

[29] K.S. Denisov, I.V. Rozhansky, N.S. Averkiev, E. Lahderanta. Phys. Rev. Lett., 117, 027202 (2016).

[30] V. Krueckl, M. Wimmer, I. Adagideli, J. Kuipers, K. Richter. Phys. Rev. Lett., 106, 146801 (2011).

[31] V.K. Dugaev, A. Crepieux, P. Bruno. Phys. Rev. B, 64, 104411 (2001).

Редактор Г.А. Оганесян 


\title{
Quantum corrections to the conductivity and anomalous Hall effect in InGaAs quantum wells with spatially separated Mn impurities
}

L.N. Oveshnikov ${ }^{1,2}$, E.I. Nekhaeva ${ }^{1,2}$

${ }^{1}$ National Research Center „Kurchatov Institute“, 123182 Moscow, Russia

2 Lebedev Physical Institute, Russian Academy of Sciences, 119991 Moscow, Russia

\begin{abstract}
The magnetotransport of heterostructures with spatial separation of magnetic Mn impurities and InGaAs quantum well was investigated. The analysis of observed weak localization effect using Hikami-Larkin-Nagaoki formula leads to abnormally low prefactor value (0.4). Obtained data indicates that inelastic $e-e$ scattering can be the dominant dephasing mechanism in structures under study. The analysis of conductivity and magnetoresistance indicates the dominant role of spin-dependent scattering on magnetic subsystem fluctuations, that is consistent with the negative magnetoresistance amplitude increase and growth of Drude conductivity upon cooling. The nature of anomalous Hall effect in studied structures is investigated, in particular, there are indications of topological contribution presence at low temperatures.
\end{abstract}

